\title{
Incidencia de la ética en la productividad a la luz de los planteamientos de García- Echevarría, Gómez-Pérez, Guédez y Morris. Estudio de caso en cuatro empresas
}

Incidence of the ethics in the productivity in the light of the approaches of García-Echevarría, Gómez-Pérez, Guédez and Morris. Case study in four companies 


\title{
Incidencia de la ética en la productividad a la luz de los planteamientos de García-Echevarría, Gómez-Pérez, Guédez y Morris. Estudio de caso en cuatro empresas ${ }^{1}$
}

\author{
Incidence of the ethics in the productivity in the light of the approaches \\ of García-Echevarría, Gómez-Pérez, Guédez and Morris. Case study in \\ four companies
}

\author{
Diego Alejandro Balcázar Gómez², Gloria Isabel Rodríguez Lozano³ \\ 2,3Universidad Nacional de Colombia, Bogotá, Colombia
}

Artículo recibido en diciembre de 2016; artículo aceptado en marzo de 2017

Citación del artículo: Balcázar, D. \& Rodríguez, L. (2017). Incidencia de la ética en la productividad a la luz de los planteamientos de García-Echevarría, Gómez-Pérez, Guédez y Morris. Estudio de caso en cuatro empresas.

I+D Revista de Investigaciones, 10(2), julio - diciembre de 2017, pp. 57 - 70

\begin{abstract}
Resumen
El objetivo de esta investigación es determinar la incidencia de la ética en la productividad de cuatro empresas a la luz de los planteamientos de García-Echevarría, Gómez-Pérez, Guédez y Morris; para lograr esto se esquematizan los planteamientos de los cuatro autores mediante un cuadro comparativo y luego se analizan aplicando la metodología Strategic Options Development and Analysis (SODA); los resultados se contrastan con los códigos de ética de cuatro empresas del medio empresarial colombiano. Además, se aplica una encuesta a los directivos y personal del área de producción de las empresas. Los resultados muestran que de los treinta valores analizados a 25 se les da una importancia media o alta, lo que se traduce en un alto grado de interiorización de los valores considerados importantes por los autores.
\end{abstract}

Palabras clave: ética, productividad, código ético.

\footnotetext{
Abstract

The objective of this research is to determine the incidence of ethics in the productivity of four companies in the light of the approaches of García-Echevarría, Gómez-Pérez, Guédez, and Morris; to achieve this, the approaches of the four authors are outlined through a comparative table and then analyzed using the Strategic Options Development and Analysis (SODA) methodology, the results are contrasted with the codes of ethics of four companies in the

1. Artículo de investigación, enfoque mixto (cualitativo y cuantitativo), resultado de un proyecto de investigación culminado perteneciente al área de Ciencias Económicas, subárea de Administración, desarrollado en la Universidad Nacional de Colombia de la ciudad de Bogotá (Colombia). Dirección: Ciudad Universitaria, Bogotá, PBX: (57) 316 5000. Fecha de inicio: 1 de febrero de 2015, fecha de terminación: 28 de febrero de 2017.

2. Ingeniero Industrial, Universidad Nacional de Colombia. Magister en Administración, Universidad Nacional de Colombia. Investigador independiente. Correo institucional: dabalcazarg@unal.edu.co.

3. Administradora de Empresas, Universidad Nacional de Colombia. PhD en Ciencias Económicas, Universidad Nacional de Colombia. Profesora Asociada de la Facultad de Ciencias Económicas de la Universidad Nacional de Colombia. Bogotá (Colombia): Dirección: Ciudad Universitaria, Edificio 311, 2 piso, Bogotá. PBX: (57) 316 5000. Correo institucional: girodriguezl@unal.edu.co.
} 
Colombian business community. In addition, survey is applied to the managers and employer of the production area of the companies. The results show that of the 30 analyzed values 25 are given a medium or high importance, which translates into a high degree of internalization of the values considered important by the authors.

Key words: ethics, productivity, ethical code.

Las empresas competitivas son éticas, pues merecen credibilidad y generan confianza (Zambrano, 2007). Y más exactamente: "... la confianza beneficia directamente a la empresa, porque aumenta la eficiencia productiva" (Cortina, 2009, p. 125).

Teniendo en cuenta que son las personas las encargadas de producir bienes y servicios en las organizaciones, cualquier mejora en la productividad debe comenzar por ellas, alineando sus necesidades individuales con las organizacionales (Cequea, Nuñez \& Rodríguez, 2011). Por ello, el comportamiento ético en las organizaciones es importante para sus integrantes, ya que estos influyen en la productividad organizacional (Cequea et al.), de tal manera que aquellos trabajadores que cuentan con una buena base ética se involucran en mayor medida en la actividad empresarial (Ballestero, Úriz \& Viscarret, 2012).

En este escenario se entiende que la ética es "rentable" para las empresas porque es un factor de innovación y un elemento diferenciador que permite proyectar a largo plazo desde los valores (Lattmann \& García, 1992). La ética empresarial es un componente imprescindible para mejorar la productividad empresarial (Val Merino, Bermúdez \& Tous, 2013).

Al respecto, Fandray (2001) sostiene que "Aunque no sea barato ni sencillo, desarrollar una cultura de la ética genera importantes beneficios para una compañía" (p. 1). Adicionalmente, Adeyeye, Adeniji, Osinbanjo \& Oludayo, (2015) explican que para que exista una alta productividad es imprescindible que todas las partes involucradas demuestren conductas éticas. Por su parte, Vimba, Coetzee \& Ukpere (2013) sostienen que las personas que aceptan la ética en sus trabajos, son más productivas.

Para Alvarado (2007) "... la tendencia a una ética grupal o individual dentro de los grupos de trabajo se veestimulada por las políticas internas de cultura organizacional, que influyen en la eficiencia y productividad de las empresas al corto y largo plazo" (p. 35).

Asimismo, como lo definen Velásquez, Núñez \& Rodríguez, (2010) uno de los aspectos que tienen mayor impacto en la productividad es la ética.

En este contexto se han escogido cuatro autores como eje central de esta investigación, por sus valiosos aportes al tema de la ética en la productividad; el resumen de sus estudios se presenta a continuación:

Santiago García-Echevarría. La productividad como magnitud de medida de los procesos económicos (1980), Empleoyproductividad(1981),Evolucióndelaproductividad y el riesgo (1981), Ética y modelos de dirección empresarial (1987), Ética empresarial y comportamientos directivos (1991), La ética en la nueva concepción institucional de la empresa (1994), Las exigencias de los procesos de cambios organizativos e institucionales. El papel de la ética (1995), Los límites de la competencia desde una perspectiva ética (1997), Ética del marketing (1999). Ética y globalización: su integración e institucionalización (Problemas éticos en torno a la globalización) (2006), La empresa en la sociedad. La dimensión ética de la empresa (2009), Cómo enseñar ética en el ámbito económico-profesional: un debate abierto (2009), La productividad de los recursos: una asignatura pendiente, educación, valores, competencias y compromiso ingredientes necesarios del recurso humano (2009), La ética de las empresas (2010), Cómo enseñar ética en el ámbito económico-empresarial. Un debate abierto (2010), La gestión ética de la economía: su configuración y articulación empresarial (2011), Incidencia del proceso educativo y de los valores humanos en la productividad laboral (2012), La dimensión ética de la economía (2015).

Rafael Gómez-Pérez. Problemas morales de la existencia humana (1982). Introducción a la ética social (1987), Ética empresarial. Teoría y casos (1990), "Notas sobre la cultura empresarial". Cuadernos de empresa y humanismo (1991), Ética. Problemas morales de la existencia humana (2000), Ética ciudadana (2007), Ética en la vida cotidiana (2010), Ética y profesión militar (2013). Vicio, virtud e hipocresía (2013).

Víctor Guédez. La ética gerencial: instrumentos estratégicos que facilitan decisiones correctas (2001), Ética, política y reconciliación (2004), Ética y práctica de la responsabilidad social. El aporte de la empresa al capital social (2006), Ser confiable. Responsabilidad social y reputación empresarial (2008), Tres visiones de la ética y responsabilidad social en la empresa del siglo XXI (junto con Emeterio Gómez e Ítalo Pizzolante, 2008), Responsabilidad social empresarial: visiones complementarias. Hacia un modelaje social (compilador, 2010).

Tom Morris. El verdadero éxito: una nueva filosofía de la excelencia (1995), Si Aristóteles dirigiera General Motors. Un 
nuevo enfoque ético de la vida empresarial (1998), Si Harry Potter dirigiera General Electric (2006), Los superhéroes y la filosofía: la verdad, la justicia y el modo socrático (2010).

Partiendo de lo anteriormente expuesto, esta investigación analiza la incidencia de la ética en la productividad de las empresas con base en los planteamientos de García-Echevarría, Gómez-Pérez, Guédez y Morris. Y buscando contrastar estos resultados con la realidad empresarial, se acude a cuatro empresas del medio industrial colombiano.

\section{Método}

Se utiliza una metodología mixta en la cual inicialmente se aplica la deducción, con algunos elementos inductivos, y se realizan análisis de tipo cuantitativo. Es importante destacar que "... la deducción permite establecer un vínculo de unión entre teoría y observación" (Dávila, 2006, p. 181).

Los aportes realizados por los cuatro autores mencionados se esquematizan en un cuadro comparativo y se analizan mediante la aplicación de la metodología Strategic options development and analysis (SODA), la cual conceptualiza diferentes puntos de vista, construyendo mapas cognitivos para modelar y registrar las visiones individuales de una situación compleja. SODA está definido como una jerarquía de conceptos relacionados por vínculos de influencia entre conceptos, medios y fines (Gutiérrez, 2012). Asimismo, proporciona una manera de identificar y organizar las preocupaciones subjetivas (Hjortsø, 2004).

Acto seguido se analizan los planteamientos de cada autor y se genera un diagrama SODA y se verifica la robustez de cada uno. Después, se contrastan para así generar un modelo completo de los cuatro; luego se analiza para verificar su robustez.

Para determinar la incidencia de la ética en la productividad de las cuatro empresas a la luz de los planteamientos de los autores escogidos se analizan los códigos de ética de las empresas contrastándolos con lo extractado mediante el diagrama SODA y el cuadro comparativo, y se aplica una encuesta a los directivos y personal del área de producción de las cuatro empresas.

\section{Planteamientos de los cuatro autores}

A continuación, se listan los documentos que se estudiaron en esta investigación por cada autor.

Santiago García-Echevarría. Management de los recursos humanos en la empresa, escrito en coautoría con Charles Lattman (1992), Ética y modelos de dirección empresarial (1987), Ética empresarial y comportamientos directivos: cómo configurar corporaciones empresariales eficientes (1991), Las exigencias de los procesos de cambios organizativos e institucionales: el papel de la ética (1995).

Rafael Gómez-Pérez. Ética empresarial: teoría y casos (1990).

Víctor Guédez. La ética gerencial: instrumentos estratégicos que facilitan decisiones correctas (2001).

Tom Morris. Si Aristóteles dirigiera General Motors. Un nuevo enfoque ético de la vida empresarial (1998).

En el Anexo 1 se presenta el cuadro comparativo de los cuatro autores.

Análisis SODA. Un mapa cognitivo SODA está definido como una herramienta de conceptos relacionados por arcos de influencia entre medios y fines. Según Henao \& Benavides (2012) se utiliza para explorar problemas complejos.

Una de las formas para realizar mapas cognitivos es representando los escritos de un individuo (Georgiuo, 2012). Para realizar esta representación, los constructos de un mapa cognitivo se conectan por medio de arcos direccionados con otros constructos, indicando un sentido de causalidad (Gutiérrez, 2012).

Adicionalmente, un mapa cognitivo exige que las afirmaciones tengan consecuencias o implicaciones, por lo que el mapa se compone de constructos (o conceptos) y flechas (o arcos) que indican la dirección de la implicación incrustada en la creencia o argumento (Eden \& Ackermann, 2004).

En la Figura 1 se muestra el diagrama SODA elaborado a partir de contrastar los cuatro diagramas SODA basados en los planteamientos de los autores. Asimismo, se estipula en el diagrama cuáles constructos corresponden a cada uno de los autores mediante las iniciales G-P (Gómez-Pérez), V-G (Víctor Guédez), T-M (Tom Morris) y G-E (García-Echevarría).

Análisis de densidad. Densidad conectiva $(D C)=\#$ arcos / \# constructos

Para este caso: $\mathrm{DC}=17 / 15=1.13$

Se esperarían proporciones de 1.15 a 1.20 para los mapas bien estructurados (Eden, 2004). De todas maneras, para Gutiérrez (2011) valores de DC por encima de 1.20 suponen redundancia de arcos. 
A partir de este resultado, se considera que el DC de este mapa es adecuado.

Análisis de dominio. “Calcula el número total de flechas que entran y salen de cada nodo, es decir su dominio inmediato" (Eden, 2004, p. 682). Explosion Grade (EG) corresponde al número total de arcos que salen de un constructo; Implosion Grade (IG) constituye el número total de arcos que entran a un constructo.
Un constructo con un relativo alto grado de dominio indica centralidad cognitiva o relevancia fundamental para la situación en cuestión. Un constructo con relativo alto EG es una causa importante y uno con IG relativamente alto indica que es percibido como un efecto importante (Georgiou \& Steaux, 2008). En la Tabla 1 se muestra el resultado de este análisis.

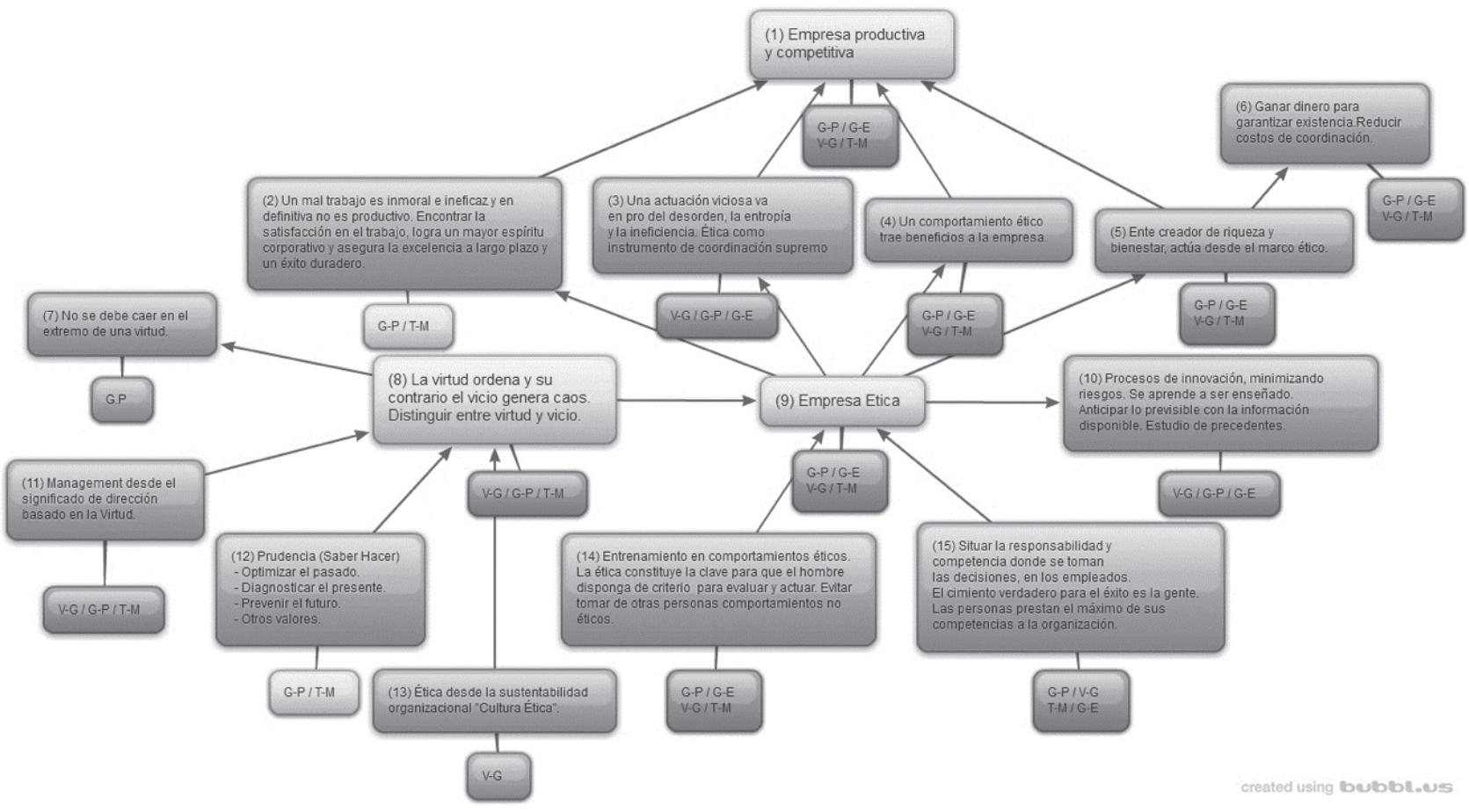

Figura 1.

DiagramaSODA

Tabla 1

Análisis de dominio

\begin{tabular}{|c|c|c|c|c|}
\hline Constructo & $\begin{array}{c}\text { EG } \\
\text { Salida } \\
\text { (explosión) }\end{array}$ & $\begin{array}{c}\text { IG } \\
\text { Entrada } \\
\text { (implosión) }\end{array}$ & $\begin{array}{c}\text { DG } \\
\text { EG+IG } \\
\text { (Grado de dominio) }\end{array}$ & Resultado \\
\hline 1 & 0 & 4 & 4 & Head \\
\hline 2 & 1 & 1 & 2 & - \\
\hline 3 & 1 & 1 & 2 & - \\
\hline 4 & 1 & 1 & 2 & - \\
\hline 5 & 2 & 1 & 3 & - \\
\hline 6 & 0 & 1 & 1 & head \\
\hline 7 & 0 & 1 & 1 & head \\
\hline 8 & 2 & 3 & 5 & $\begin{array}{l}\text { Consecuencia } \\
\text { significante }\end{array}$ \\
\hline 9 & 5 & 3 & 8 & Causa significante \\
\hline 10 & 0 & 1 & 1 & C \\
\hline 11 & 1 & 0 & 1 & Tail \\
\hline 12 & 1 & 0 & 1 & Tail \\
\hline 13 & 1 & 0 & 1 & Tail \\
\hline 14 & 1 & 0 & 1 & Tail \\
\hline 15 & 1 & 0 & 1 & Tail \\
\hline
\end{tabular}

Fuente: Autores 
Se observa que el constructo \#8, “La virtud ordena, el vicio caotiza", corresponde a una consecuencia significante fundamentada en los valores, el management orientado en direccionar desde la virtud y en la ética, y desde la sustentabilidad organizacional por medio de una cultura ética. Mientras que el constructo \#9, "Una empresa ética", corresponde a una causa significante. Este último también es una causa mayor con significado estructural en el mapa, que afecta los procesos y los resultados en la organización que derivan en lograr una empresa productiva y competitiva. Este último constructo también tiene el más alto DG, mostrando una centralidad cognitiva en este tema desde las percepciones de los autores.

\section{Análisis de heads and tails}

Este análisis de complejidad tiene en cuenta la relación del número de heads (cabezas) y el número de tails (colas), respecto al número total de nodos.

Como puede observarse en la Tabla 1, el objetivo principal o head corresponde al constructo \#1 "Empresa productiva y competitiva", asimismo aparece un par adicional de objetivos que consisten en lograr que la empresa mantenga su foco de ganar dinero para garantizar la existencia (\#6) y que no se caiga en el extremo de una virtud (\#7) que puede llegar ser perjudicial para lograr los objetivos.

En cuanto a tails, están el entrenamiento en comportamientos éticos (\#14), en lograr situar la responsabilidad y la competencia en los empleados de la base (\#15), trabajo desde los valores (\#12), las virtudes (\#11), el liderazgo desde la virtud y una cultura ética a todos los niveles de la organización (\#13). Por lo cual, si se quiere alcanzar el objetivo, que en este caso es lograr una empresa productiva y competitiva, se deben tener en cuenta estas causas primarias ya que influyen en el proceso de toma de decisiones.

\section{Conclusiones SODA}

Por lo anterior, se establece que, por medio de la conceptualización de los planteamientos de los autores, se llega a la conclusión que una empresa ética es una causa significante de una empresa productiva y competitiva. Es importante soportarla en causas primarias como el entrenamiento en comportamientos éticos, en situar la responsabilidad en los empleados, en un trabajo basado en los valores y en una cultura ética a todos los niveles de la organización. Asimismo, además de lograr una empresa productiva y competitiva, la ética tiene como consecuencia significativa lograr en las personas una actuación basada en virtudes que genera orden en el interior de la empresa, disminuyendo la complejidad organizativa y facilitando los procesos.

\section{Las empresas}

Empresa 1. Es considerada una de las primeras compañías mundiales en productos de marcas de consumo masivo. Hace presencia en Colombia desde 1948. Desde el 2004, esta empresa viene desplegando su actual misión, que se lleva a cabo con el lema "Aportar vitalidad a la vida".

Empresa 2. Es una empresa colombiana de talla internacional, cuenta en su portafolio con una amplia gama de productos en la línea de alimentos y aseo. Es una empresa de tradición, pero también moderna y futurista; ya que desde 1906 está en Colombia, pero tiene una gran presencia en mercados internacionales y de consumo fuera del hogar.

Empresa 3. Esta empresa hace parte del grupo fabricante de envases de vidrio más grande del mundo y es socio preferido de muchas de las marcas líderes de alimentos y bebidas a nivel mundial. En Colombia está presente con cuatro plantas de producción.

Empresa 4. La operación más grande de la empresa en Latinoamérica se encuentra en Colombia, y cuenta con trece marcas en la categoría de bebidas, producidas en ocho plantas, además, una fábrica de etiquetas y una de tapas. Más de 4100 colaboradores hacen parte de la empresa de manera directa, y unas 3500 personas adicionales de forma indirecta.

\section{Resultados}

\section{Revisión códigos de ética}

En el sentido estricto de comparar los códigos de ética respecto a los planteamientos de los autores se encuentra que ninguno está alineado por completo y que cada código de ética es diferente. En la Tabla 2, se esbozan algunas diferencias y similitudes, sombreando los puntos que concuerdan respecto a 21 premisas, tomando en conjunto los 4 códigos de ética. Estas 21 premisas se construyen con base en el cuadro comparativo entre autores (Anexo 1) numerales 5, 8, 9 y 10. 
Tabla 2

Diferencias y similitudes en los códigos de ética

Empresa 1 Empresa 2 Empresa 3 Empresa 4

1 Se considera que por medio de la ética se puede generar una buena reputación en los negocios.

2 Se da importancia a la ética dentro de la organización desde el enfoque de mejora en la productividad.

3 Se tiene en cuenta el impacto que tiene la ética en la competitividad de las organizaciones.

4 Se da mayor o igual importancia a las relaciones interpersonales que las relaciones comerciales.

5 Se entiende que el comportamiento ético y moral es instintivo y no solo depende de normas, órdenes y sistemas.

6 Se tiene en cuenta que la falta de ética implica incremento en la complejidad organizativa.

7 Se da importancia al proceso de innovación como factor clave para minimizar los riesgos éticos.

8 Se tienen en cuenta los valores definidos por los autores con un alto grado de significado, para lograr organizaciones éticas.

9 Se tiene en cuenta el peligro de caer en los extremos de una virtud.

10 Se hace mención de temas como la motivación del personal, la importancia en que el personal conozca el trabajo que realiza y el reconocimiento a los logros del personal.

11 Se reconoce la importancia de un liderazgo íntegro, en donde la gerencia tiene un papel fundamental.

12 Se aclara que las empresas que mantienen altos estándares de conducta ética tienen una ventaja competitiva.

13 Se resalta que el código de ética es solo una guía.

14 Se busca un entorno laboral en el que se estimulan y esperan conversaciones constructivas y abiertas.

15 Se plantea al menos un instrumento como hoja de ruta para la toma de decisiones éticas.

16 Se propende por mantener un entorno de trabajo donde los empleados se sientan valorados, apoyados y facultados.

17 Se plantean mecanismos para mantener un entrenamiento en comportamientos éticos.

18 Se espera un trato hacia a las personas con respeto, y no existe el acoso ni la discriminación en el lugar de trabajo.

19 Cada persona tiene la responsabilidad personal de modelar conductas que contribuyan con un entorno de trabajo seguro y productivo.

20 La empresa se compromete a proporcionar un entorno laboral saludable y seguro a todos sus empleados.

21 Se da importancia a la justicia desde el punto de vista que todos los empleados se deben esforzar por tratarse de forma justa.
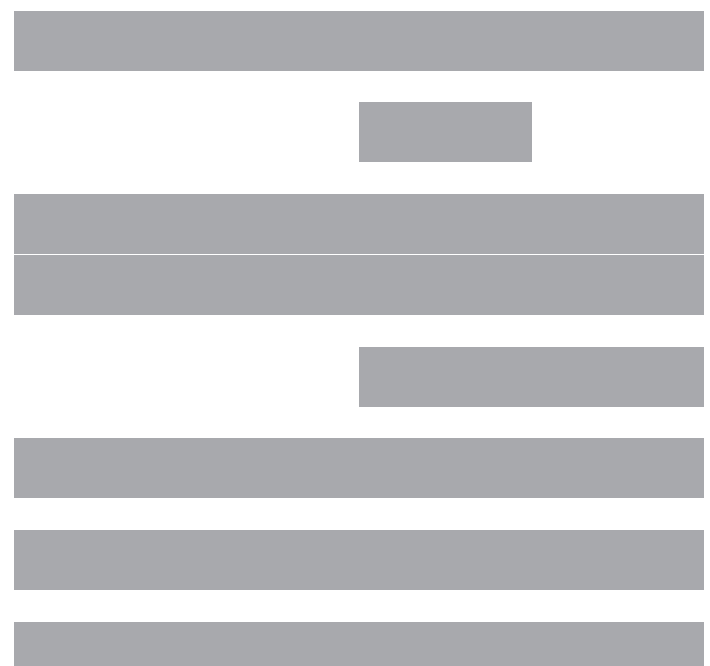

$$
\text { s }
$$

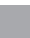
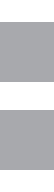

\section{Fuente: Autores}




\section{Comparativo de valores}

En la Tabla 3 se presenta el comparativo con los valores definidos por uno o varios de los cuatro autores en relación con el tema de impacto de la ética en la productividad que fueron enunciados anteriormente en el cuadro comparativo entre autores (Anexo 1), numerales 6 y 7.

Posteriormente, se comparan con los valores en los que se basan cada uno de los códigos de ética. Sin embargo, no se espera que un código de ética contemple todos los valores, ya que es solo una guía. Se considera que un código de ética es un documento formal, escrito y distinguible, que consiste en normas morales utilizadas para guiar al empleado o el comportamiento empresarial (Schwartz, 2011).
Con el fin de identificar si un valor es común entre una empresa y los autores, se utiliza el siguiente código de colores:

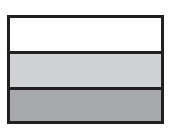

Valores no contemplados Valores en común

Diferencia entre autores y códigos de ética

Adicionalmente, para los valores en común, se identifican qué autores los definen como importantes colocando la inicial del autor, tal como se utilizó para el diagrama SODA; para los valores que son comunes a los cuatro autores se coloca la palabra "TODOS".

Tabla 3

Comparativo con los valores definidos por uno o varios de los cuatro autores

\begin{tabular}{|c|c|c|c|c|c|}
\hline Valores & Autores & Empresa 1 & Empresa 2 & Empresa 3 & Empresa 4 \\
\hline Honestidad & (V-G / T-M) & & & & \\
\hline Respeto & (V-G / T-M) & & & & \\
\hline Confianza & (TODOS) & & & & \\
\hline Integridad & (V-G / T-M) & & & & \\
\hline \multicolumn{6}{|l|}{ Reputación } \\
\hline Responsabilidad & (TODOS) & & & & \\
\hline Transparencia & $(\mathrm{V}-\mathrm{G})$ & & & & \\
\hline Justicia & (G-P / T-M) & & & & \\
\hline Compromiso & (T-M) & & & & \\
\hline Prudencia & (G-P / V-G / T-M) & & & & \\
\hline Lealtad & (V-G / T-M) & & & & \\
\hline Solidaridad & $(\mathrm{V}-\mathrm{G})$ & & & & \\
\hline Perseverancia & (G-P) & & & & \\
\hline \multicolumn{6}{|l|}{ Sencillez } \\
\hline \multicolumn{6}{|l|}{ Innovación } \\
\hline \multicolumn{6}{|l|}{ Inspiración } \\
\hline \multicolumn{6}{|l|}{ Valentía } \\
\hline \multicolumn{6}{|l|}{ Dignidad } \\
\hline \multicolumn{6}{|l|}{ Cumplimiento } \\
\hline \multicolumn{6}{|l|}{ Equidad } \\
\hline \multicolumn{6}{|l|}{ Decencia } \\
\hline \multicolumn{6}{|l|}{ Orgullo } \\
\hline Excelencia & & & & & \\
\hline
\end{tabular}

Fuente: Autores 
Hay doce valores en común entre los autores y los códigos de ética. Asimismo, hay valores que contemplan los autores, pero no las empresas: competitividad, fortaleza, generosidad, sobriedad, flexibilidad, reconocimiento, empatía, autoestima, comprensión, autorresponsabilidad, compasión, tolerancia, compañerismo, amabilidad, sensibilidad, sabiduría, paciencia y cortesía.

Los valores organizacionales proporcionan un sentido de dirección común para todos los empleados y establecen directrices para su compromiso diario, además de promover el logro de los resultados. De esta forma, el sistema de valores de una organización tiene efecto en la productividad y establece un punto de partida para la mejora (Velásquez, Rodríguez \& Guaita, 2012).

Por lo tanto, los valores adquieren importancia al indicar cómo deben comportarse los miembros de una organización con el fin de alcanzar sus objetivos. Aquí, los valores inciden en el objetivo de las empresas de mejorar la productividad, y de acuerdo a los planteamientos de los cuatro autores hay valores que tienen un mayor efecto.

Es de destacar que las organizaciones ven la "reputación" como un valor en sí, mientras que los autores la consideran una consecuencia de actuar éticamente. Esto indicaría que existe una brecha entre la realidad de las organizaciones y los valores que identifican los cuatro autores, por lo cual es importante que las empresas se esfuercen por poner en práctica los valores faltantes en sus filosofías de gestión. De esta manera lograrían impactar positivamente en la productividad, la competitividad y la calidad, y por lo tanto, en la excelencia duradera de las organizaciones.

\section{Aplicación de la encuesta}

Por ser el método más recomendado para acopiar información de este tipo (Fink, 2006) se realizó una encuesta a los directivos y personal del área de producción de las empresas.

La encuesta fue diseñada utilizando la escala Likert, destinada a medir actitudes y predisposiciones individuales a actuar de cierta manera en contextos sociales específicos (García, Aguilera \& Castillo, 2011).

Entre los aspectos constitutivos de la escala Likert es importante resaltar las alternativas que corresponden a las opciones de respuesta. Para conseguir la puntuación se suman los valores obtenidos para cada pregunta (Malave, 2007). “Lo más usual es asignar una ponderación arbitraria, en la cual se pueden usar los números 1, 2, 3, 4, 5" (García, Aguilera \& Castillo, 2011, p. 9), que se utilizará en este caso. Cada uno de los puntos de la encuesta se calificó de 1 a 5 , siendo 5 el puntaje que indica un mayor sentido de orientación hacia la ética.

La encuesta está compuesta de dos preguntas introductorias y seis segmentos donde se agrupan los conceptos de los cuatro autores. Estos seis segmentos buscan conocer, a grandes rasgos, si el encuestado:

1. Entiende que la ética está relacionada con la empresa y representa beneficios.

2. Considera que la ética es un factor estratégico en las organizaciones.

3. Razona que la ética impacta en la productividad y en el éxito de las empresas.

4. Identifica los factores de éxito de la ética en las organizaciones.

5. Acepta las acciones para mantener y consolidar la ética al interior de las organizaciones.

6. Da importancia a los valores considerados significativos por los cuatro autores.

Los resultados que se muestran en la Tabla 4 se obtuvieron tras compilar y calificar los datos de la encuesta.

\section{Tabla 4}

Resultado de la encuesta

\begin{tabular}{lcccc}
\hline Proyecto & Empresa 1 & Empresa 2 & Empresa 3 & Empresa 4 \\
\hline Grupo 1 & $95 \%$ & $100 \%$ & $65 \%$ & $100 \%$ \\
Grupo 2 & $87 \%$ & $100 \%$ & $100 \%$ & $97 \%$ \\
Grupo 3 & $89 \%$ & $100 \%$ & $100 \%$ & $94 \%$ \\
Grupo 4 & $90 \%$ & $100 \%$ & $100 \%$ & $96 \%$ \\
Grupo 5 & $91 \%$ & $100 \%$ & $97 \%$ & $91 \%$ \\
TOTAL & $90,0 \%$ & $100,0 \%$ & $95,7 \%$ & $94,3 \%$ \\
\end{tabular}

Fuente: Autores

\section{Resultados del sexto segmento}

De acuerdo con el resultado obtenido, los valores de menor importancia son la fortaleza, el reconocimiento, la solidaridad, la compasión y la cortesía, y los de mayor importancia son prudencia (saber hacer), honestidad, lealtad, confianza, integridad, respeto, generosidad, 
empatía, autoestima, autorresponsabilidad, tolerancia, transparencia, sabiduría, paciencia y justicia.

Es de resaltar que de los valores analizados a veinticinco se les da una importancia media o alta, lo que se traduce en un alto grado de interiorización de los valores considerados importantes por los autores, y aunque como se vio anteriormente, en los códigos de ética solo se contemplan doce, luego de la encuesta se evidencia que a pesar de no estar en los códigos de ética los encuestados los consideran importantes, corroborando la premisa de que un código de ética es solo una guía, por lo cual no se espera que contemple todos los valores.

De lo anterior se puede concluir que las personas encuestadas en cada una de las empresas tienen una percepción favorable de la importancia de la ética y su impacto en la productividad. Sin embargo, aún hay brechas que cerrar, pues haber obtenido respuestas como que la ética en lo económico no tiene sentido o que la ética son limitaciones y restricciones, o que los números y los proyectos son más importantes que las personas, es un claro indicio de que bajo ciertas circunstancias se puede llegar a desestimar la ética.

Asimismo, se llegó a la conclusión de que si, en definitiva, las empresas quieren impactar en la productividad por medio de la ética, deben realizar cambios para que esta se convierta en un pilar estratégico en el cual las personas sean el cimiento de la organización, entendiendo que la ética y la productividad están interrelacionadas y en donde continuamente se apliquen instrumentos para reforzar los comportamientos éticos.

\section{Conclusiones y recomendaciones}

Como resultado de esta investigación es posible concluir que, de acuerdo a los planteamientos de GarcíaEchevarría, Gómez-Pérez, Guédez y Morris, la ética incide en la productividad de las cuatro empresas, como se reafirma a continuación.

Desde la perspectiva económica y social de García-Echevarría la ética y la productividad están intrínsecamente relacionadas, y esta última es uno de los parámetros más importantes de la competitividad. La ética influye entonces como principio de coordinación con el fin de alinear los comportamientos de los individuos y la organización para lograr el uso eficiente de los recursos disponibles. Por lo tanto, en la medida que los comportamientos estén alineados con lo que busca la organización, la ética disminuye la complejidad organizativa, aumentando la productividad y la capacidad competitiva de la organización.
Por su parte, para Gómez-Pérez la ética y la productividad están estrechamente relacionadas, en tanto que un entorno ético asegura una alta productividad en el trabajo realizado, pues la ética impacta en la motivación del personal asegurando que las personas hagan un buen trabajo, eficaz y productivo. De esta manera, la ética constituye un rasgo competitivo en la organización.

Para Guédez la ética es, en definitiva, una inversión beneficiosa para las organizaciones, ya que además de incrementar la productividad y la calidad, las hace sustentables en el tiempo. Y aunque en la mayoría de los casos es más fácil no ser ético, vale la pena asumir ese desafío en las tareas de producción de riqueza, pues se generan sinergias positivas para la organización.

Finalmente, Morris estima que existe una importante conexión entre la ética y la productividad, entendiendo la ética desde las cuatro dimensiones de la experiencia humana, la verdad, la belleza, la bondad y la unidad. Si alguna de estas falta se tendrá como resultado falta de motivación, baja productividad, falta de creatividad y otros aspectos no deseados.

Ahora bien, según los autores, en un principio la ética se percibía como algo ajeno a las organizaciones; sin embargo, en la actualidad cobra importancia como un asunto de inteligencia empresarial, además la ética empresarial tiene una naturaleza estratégica, ya que se vincula con la sobrevivencia de las empresas y la convivencia de las personas.

Luego de aplicar la metodología SODA se estableció que una empresa ética es una causa significante de una empresa productiva y competitiva, con lo cual se valida que la ética impacta en la productividad de las organizaciones. Sin embargo, es importante soportarla en causas primarias como el entrenamiento en comportamientos éticos, en situar la responsabilidad en los empleados, en un trabajo basado en los valores y en una cultura ética en todos los niveles de la organización. Adicionalmente, la ética tiene como consecuencia significante lograr en las personas una actuación basada en virtudes que genera orden en el interior de la empresa, disminuyendo la complejidad organizativa y facilitando los procesos.

Sin embargo, a pesar del grado de significación de la ética en la empresa como una causal de la productividad, en los códigos de ética no se aborda el tema y solo en uno de los códigos (Empresa 3) se hace una breve referencia sobre su impacto en la competitividad. Por ende, en lo que refiere a los códigos de ética, se observa que las empresas no dimensionan la importancia de la ética para 
impactar en su productividad. Esto no quiere decir que las empresas no le den importancia a la ética, la diferencia es que el enfoque se da en mayor medida en proteger la reputación y en asegurar las relaciones comerciales, así como en evitar prácticas como el acoso laboral, la discriminación, el soborno, el tráfico de influencias y el conflicto de intereses, entre otros.

Del mismo modo, los valores de las empresas, que adquieren importancia al indicar cómo deben comportarse los miembros de una organización con el fin de alcanzar los objetivos, pueden llegar a incidir en el objetivo de las empresas de mejorar la productividad.

De acuerdo a los planteamientos de los cuatro autores se establecen una serie de valores a tener en cuenta para impactar en la productividad, de los cuales doce son comunes a una o varias empresas de acuerdo a los códigos de ética; estos son, a saber: honestidad, respeto, confianza, integridad, responsabilidad, transparencia, justicia, compromiso, prudencia, lealtad, solidaridad y perseverancia.

Estos doce valores comunes establecen un punto de partida para mejorar la productividad, por lo cual sería significativo ampliar en futuros trabajos los modos de actuación de estos valores y evaluar sus consecuencias en los indicadores de productividad.

Según las encuestas, a 25 valores se les da una importancia media o alta, lo que se traduce en un alto grado de interiorización de los valores considerados importantes por los autores, y aunque, como se vio anteriormente en los códigos de ética, solo se contemplan 12 de los 30, tras procesar los resultados de la encuesta se evidencia que, a pesar de no estar en los códigos de ética, los encuestados los consideran importantes.

Existe una oportunidad de mejora entre la realidad de las cuatro organizaciones y los valores que identifican los cuatro autores, por lo cual es importante que las empresas se esfuercen por poner en práctica los valores faltantes en sus filosofías de gestión, y en consolidar los valores que, aunque no se declaren en los códigos de ética, sí son contemplados por sus empleados. Con ello se lograría impactar positivamente en la productividad, la competitividad y la calidad, y, por lo tanto, en la excelencia duradera de las organizaciones.

Las personas encuestadas en cada una de las empresas tienen una percepción favorable de la importancia de la ética y su impacto en la productividad. Sin embargo, aún hay brechas que cerrar; esto indica un bajo grado de madurez en las empresas desde la perspectiva de la ética como una de las causales de la mejora en la productividad y competitividad de las organizaciones.

Por lo anterior, se recomienda a las empresas hacer de la ética un pilar que permita mejorar la productividad con base en los postulados de los cuatro autores, que trabajen de la mano con otras iniciativas para la obtención de mejores resultados y el cumplimiento de los objetivos. En donde la moral y la ética vayan más allá de las normas, basándose en las virtudes y los valores de los individuos, desde el liderazgo ético de la gerencia, definiendo reglas claras y comportamientos transparentes. De esta manera se logra la realización de las personas y por lo tanto, la mejora en la eficiencia y la productividad.

Se deben realizar cambios para que la ética se convierta en un pilar estratégico en el cual las personas sean el cimiento de la organización, entendiendo que la ética y la productividad están interrelacionadas y aplicando, continuamente, instrumentos para reforzar los comportamientos éticos.

\section{Referencias}

Adeyeye, J., Adeniji, A., Osinbanjo, A.\& Oludayo, O. (2015). Effects of workplace ethics on employees and organisational productivity in Nigeria. International Conference on African Development Issues. Tokyo: OSAA.

Alvarado, D. (2007). La ética del trabajo y su influencia en la eficiencia y productividad de las pequeñas organizaciones laborales. Revista Faces, 18(1), 35-51.

Ballestero, A., Úriz, M. \& Viscarret, J. (2012). Dilemas éticos de las trabajadoras y los trabajadores sociales en España. Papers, 97(4), pp. 875-898.

Cequea, M., Nuñez, M. \& Rodríguez, C. (2011). Factores grupales que inciden en la productividad. Universidad, Ciencia y Tecnología, 15(61), 223-231.

Cortina, A. (2009). Ética de la empresa: no solo responsabilidad social. Revista Portuguesa de Filosofia, 65, 113-127.

Dávila, G. (2006). El razonamiento inductivo y deductivo dentro del proceso investigativo en ciencias experimentales y sociales. Laurus, 12, 180-205.

Eden, C. (2004). Analyzing cognitive maps to help structure issues or problems. European Journal of Operational Research, 159, 673-686.

Eden, C. \& Ackermann, F. (2004). Cognitive mapping expert views for policy analysis in the public sector. European Journal of Operational Research,152, 615-630. 
Fandray, D. (2001). El compromiso con los valores. Workforce Magazine, 6, 1-3.

Fink, A. (2006). How to conduct surveys. A step by step guide. Thousand Oaks: SAGE Publications.

García, S., Aguilera, J.\& Castillo, A. (2011). Guía técnica para la construcción de escalas de actitud. Odiseo. Revista Electrónica de Pedagogía,16, 1-13.

García, S. (1980). La productividad como magnitud de medida de los procesos económicos. Alcalá de Henares: Instituto de Dirección y Organización de Empresas, Universidad de Alcalá.

García, S. (1981a). Empleo y productividad. Alcalá de Henares: Instituto de Dirección y Organización de Empresas, Universidad de Alcalá.

García, S. (1981b). Evolución de la productividad y el riesgo. Alcalá de Henares: Instituto de Dirección y Organización de Empresas, Universidad de Alcalá.

García, S. (1987). Ética y modelos de dirección empresarial. Alcalá de Henares: Instituto de Dirección y Organización de Empresas, Universidad de Alcalá.

García, S. (1991). Ética empresarial y comportamientos directivos: cómo configurar corporaciones empresariales eficientes. Alcalá de Henares: Instituto de Dirección y Organización de Empresas, Universidad de Alcalá.

García, S. (1994). La ética en la nueva concepción institucional de la empresa. Alcalá de Henares: Instituto de Dirección y Organización de Empresas, Universidad de Alcalá.

García, S. (1995). Las exigencias de los procesos de cambios organizativos e institucionales : el papel de la ética. Alcalá de Henares: Instituto de Dirección y Organización de Empresas, Universidad de Alcalá.

García, S. (1997). Los límites de la competencia desde una perspectiva ética. Alcalá de Henares: Instituto de Dirección y Organización de Empresas, Universidad de Alcalá.

García, S. (1999). Ética del marketing. Alcalá de Henares: Instituto de Dirección y Organización de Empresas, Universidad de Alcalá.

García, S. (2006). Ética y globalización: su integración e institucionalización (problemas éticos en torno a la globalización). Alcalá de Henares: Instituto de Dirección y Organización de Empresas, Universidad de Alcalá.

García, S. (2009a). Cómo enseñar ética en el ámbito económicoprofesional: un debate abierto. Alcalá de Henares: Instituto de Dirección y Organización de Empresas, Universidad de Alcalá.

García, S. (2009b). La dimensión ética de la empresa. Alcalá de Henares: Instituto de Dirección y Organización de Empresas, Universidad de Alcalá.

García, S. (2009c). La productividad de los recursos: una asignatura pendiente, educación, valores, competencias y compromiso ingredientes necesarios del recurso humano. Alcalá de Henares: Instituto de Dirección y Organización de Empresas, Universidad de Alcalá.

García, S. (2010a). Cómo enseñar ética en el ámbito económicoempresarial. Un debate abierto. Alcalá de Henares: Instituto de Dirección y Organización de Empresas, Universidad de Alcalá.

García, S. (2010b). La ética de las empresas. Alcalá de Henares: Instituto de Dirección y Organización de Empresas, Universidad de Alcalá.

García, S. (2011). La gestión ética de la economía: su configuración y articulación empresarial. Alcalá de Henares: Instituto de Dirección y Organización de Empresas, Universidad de Alcalá.

García, S. (2012). Incidencia del proceso educativo y de los valores humanos en la productividad laboral. Conferencias y trabajos de investigación del Instituto de Dirección y Organización de Empresas, (346), 1-29.

García, S. (2015). La dimensión ética de la economía. Alcalá de Henares: Instituto de Dirección y Organización de Empresas, Universidad de Alcalá.

Georgiou, I. \& Steaux, P. (2008). Strategic options development and analysis: the case of the brazilian railways. Rio de Janeiro: Fundação Getulio Vargas.

Georgiuo, I. (2012). Messing about in transformations: structured systemic planning for systemic solutions to systemic problems. European Journal of Operational Research,223, 392-406.

Gómez, R. (1982). Problemas morales de la existencia humana. Madrid: Ediciones Casals.

Gómez, R. (1987). Introducción a la ética social. Madrid : Ediciones Rialp.

Gómez, R. (1990). Ética empresarial: teoría y casos. Madrid: Ediciones Rialp. 
Gómez, R. (1991). Notas sobre la cultura empresarial. Cuadernos de Empresa y Humanismo. Cuaderno 27, 3-23.

Gómez, R. (2000). Ética. Problemas Morales de la Existencia Humana. El Magisterio español.

Gómez, R. (2007). Ética ciudadana. Sekotia.

Gómez, R. (2010). Ética en la vida cotidiana. Romana.

Gómez, R. (2013a). Ética y profesión militar. Rialp.

Gómez, R. (2013b). Vicio, virtud e hipocresía. Rialp.

Guédez, V. (2001). La Ética gerencial: instrumentos estratégicos que facilitan decisiones correctas. Caracas: Planeta.

Guédez, V. (2004). Ética, política y reconciliación. Criteria.

Guédez, V. (2006). Ética y práctica de la Responsabilidad social. El aporte de la empresa al capital social. Planeta.

Guédez, V. (2008a). Ser confiable. Responsabilidad social y reputación empresarial. Planeta.

Guédez, V. (2008b). Tres visiones de la ética y responsabilidad social en la empresa del siglo XXI. Planeta.

Guédez, V. (2010). Responsabilidad Social Empresarial: visiones complementarias. Hacia un modelaje social (comp.). Alianza Social de Venamcham.

Gutiérrez, R. (2011). Strategic options development and analysis (SODA) - Análisis Parte 2. Recuperado 3 de marzo, 2016, de http://www.fcmfmpep.org.br/disciplinas/turma2/MB-726/ Aula02/Aula SODA II espanhol.ppt

Gutiérrez, R. (2012). Mapa cognitivo do método SODA aplicado ao problema da produção científica no ensino superior. Recuperado 23 de febrero, 2016, de http://www.epio.org. ar/attachments/126_Seminario\%20Belderrain\%20-\%20 Castellini.pdf

Henao, F. \& Benavides J. (2012). Una aproximación multimetodológica para la definición de portafolios de proyectos de inversión. Academia. Revista Latinoamericana de Administración, 50, 79-99.

Hjortsø, C. (2004). Enhancing public participation in natural resource management using Soft OR: an application of strategic option development and analysis in tactical forest planning. European Journal of Operational Research,152, 667-683.
Lattmann, C., \& García, S. (1992). Management de los Recursos Humanos en la empresa. Madrid: Díaz de Santos.

Malave, N. (2007). Trabajo modelo para enfoques de investigación acción participativa programas nacionales de formación. Escala tipo Likert. Recuperado 4 de febrero, 2016, de http://uptparia.edu.ve/documentos/ F\%C3\%ADsico\%20de\%20Escala\%20Likert.pdf

Morris, T. (1995). El verdadero éxito: una nueva filosofía de la excelencia. Planeta.

Morris, T. (1998). Si Aristóteles dirigiera General Motors. Un nuevo enfoque ético de la vida empresarial. Bogotá: Planeta.

Morris, T. (2006). Si Harry Potter dirigiera General Electric. Planeta.

Morris, T. (2010). Los superhéroes y la filosofía: la verdad, la justicia y el modo socrático. Planeta.

Schwartz, M. (2011). The nature of the relationship beetwen corporate codes of ethics and behaviour. Journal of Business Ethics, 32, 247-262.

Val Merino, C., Bermúdez, G., y Tous, D. (2013). La ética como generadora de confianza y crecimiento en tiempos de crisis en las pyme del sector TIC de Madrid. Revista de Estudios Empresariales, 1, 6-20.

Velásquez, Y., Rodríguez, C. \& Guaita, W. (2012). Los valores organizacionales: referencia para la evaluación de la productividad. 6th International Conference on Industrial Engineering and Industrial Management XVI. Congreso de Ingeniería de Organización, Vigo.

Vimba, M., Coetzee, J. \& Ukpere, W. (2013). A relationship between leadership work ethic and organisational performance in the public sector. J Soc Sci, 35(2), 169-180.

Zambrano, E. (2007). El valor de la ética para alcanzar la excelencia en la gerencia universitaria. Negotium / Ciencias Gerenciales, 8, 30-63. 


\section{ANEXO 1}

\section{Cuadro comparativo}

\begin{tabular}{|c|c|c|c|c|c|}
\hline & & $\begin{array}{c}\text { Rafael } \\
\text { Gomez-Pérez }\end{array}$ & Víctor Guédez & Tom Morris & $\begin{array}{c}\text { Santiago } \\
\text { Garcia-Echevarría }\end{array}$ \\
\hline 1 & $\begin{array}{l}\text { Empresa antes } \\
\text { ética }\end{array}$ & $\begin{array}{l}\text { Sistema cerrado, solo lo } \\
\text { estrictamente necesario. La } \\
\text { ética no tiene importancia. }\end{array}$ & $\begin{array}{l}\text { El concepto de ética en la } \\
\text { empresa es excluyente e } \\
\text { irreconciliable. }\end{array}$ & $\begin{array}{l}\text { La ética como limitaciones y } \\
\text { restricciones, que no } \\
\text { permiten hacer lo que se } \\
\text { quiere. }\end{array}$ & $\begin{array}{l}\text { La ética en lo económico no } \\
\text { tiene sentido. }\end{array}$ \\
\hline 2 & $\begin{array}{l}\text { Empresa ahora } \\
\text { ética }\end{array}$ & $\begin{array}{l}\text { Ente creador de riqueza y } \\
\text { bienestar, actúa desde el } \\
\text { marco ético. }\end{array}$ & $\begin{array}{l}\text { La ética empresarial tiene } \\
\text { una naturaleza estratégica, } \\
\text { ya que se vincula con la } \\
\text { sobrevivencia de las } \\
\text { empresas y la convivencia } \\
\text { de las personas. }\end{array}$ & $\begin{array}{l}\text { Redescubrir la importancia } \\
\text { vital de la calidad del } \\
\text { producto y el servicio, } \\
\text { buscando realizar el trabajo } \\
\text { para lograr más eficiencia y } \\
\text { nuevas formas de } \\
\text { excelencia. }\end{array}$ & $\begin{array}{l}\text { La ética constituye la clave } \\
\text { para que el hombre } \\
\text { disponga de criterio para } \\
\text { evaluar y actuar. }\end{array}$ \\
\hline 3 & $\begin{array}{l}\text { Fundamento } \\
\text { (finalidad) de la } \\
\text { empresa }\end{array}$ & $\begin{array}{l}\text { Ganar dinero para garantizar } \\
\text { existencia. }\end{array}$ & $\begin{array}{l}\text { Producción de riqueza y } \\
\text { utilidad social. }\end{array}$ & $\begin{array}{l}\text { Éxito financiero en un } \\
\text { mundo competitivo. }\end{array}$ & $\begin{array}{l}\text { Reducir costos de } \\
\text { coordinación. }\end{array}$ \\
\hline 4 & $\begin{array}{l}\text { Planteamiento } \\
\text { inicial del autor }\end{array}$ & $\begin{array}{l}\text { Management desde el } \\
\text { significado de dirección } \\
\text { basado en la Virtud. }\end{array}$ & $\begin{array}{l}\text { Ética desde la } \\
\text { sustentabilidad } \\
\text { organizacional "cultura } \\
\text { ética". } \\
\text { Factor conocimiento. } \\
\text { Factor emocional. } \\
\text { Factor ético. }\end{array}$ & $\begin{array}{l}\text { Excelencia humana } \\
\text { sostenible basada en la } \\
\text { verdad, la belleza, la bondad } \\
\text { y la unidad. }\end{array}$ & $\begin{array}{l}\text { Ética como instrumento de } \\
\text { coordinación supremo, } \\
\text { mediante el logro de bajos } \\
\text { costos de coordinación, } \\
\text { garantizando el éxito de la } \\
\text { organización. }\end{array}$ \\
\hline 5 & $\begin{array}{l}\text { Detalle sobre el } \\
\text { planteamiento } \\
\text { del autor }\end{array}$ & $\begin{array}{l}\text { La virtud ordena y su } \\
\text { contrario el vicio genera } \\
\text { caos. } \\
\text { No se debe caer en el } \\
\text { extremo de una virtud. }\end{array}$ & $\begin{array}{l}\text { La ética es un factor clave } \\
\text { para el liderazgo como } \\
\text { trabajo conjunto, fuente de } \\
\text { visión compartida y energía } \\
\text { para el logro. }\end{array}$ & $\begin{array}{l}\text { Se deben hallar los } \\
\text { cimientos para encontrar la } \\
\text { satisfacción en el trabajo. }\end{array}$ & $\begin{array}{l}\text { El individuo es portador de } \\
\text { una determinada forma de } \\
\text { conducta y conjunto de } \\
\text { normas éticas. }\end{array}$ \\
\hline 6 & $\begin{array}{l}\text { Principales } \\
\text { Valores } \\
\text { definidos por el } \\
\text { autor }\end{array}$ & $\begin{array}{l}\text { Prudencia (saber hacer): } \\
\text { Optimizar el pasado. } \\
\text { Diagnosticar el presente. } \\
\text { Prevenir el futuro. }\end{array}$ & $\begin{array}{l}\text { Prudencia (saber hacer), } \\
\text { Honestidad, lealtad. }\end{array}$ & $\begin{array}{l}\text { Verdad (honestidad). } \\
\text { Belleza (ambiente } \\
\text { adecuado). } \\
\text { Bondad (hacerlo bien, } \\
\text { haciendo el bien). Unidad }\end{array}$ & $\begin{array}{l}\text { Responsabilidad, } \\
\text { competitividad. } \\
\text { Flexibilidad: } \\
\text { confianza. }\end{array}$ \\
\hline 7 & $\begin{array}{l}\text { Otras Valores } \\
\text { relevantes en } \\
\text { una empresa } \\
\text { ética }\end{array}$ & $\begin{array}{l}\text { Fortaleza o firmeza: } \\
\text { Paciencia, Perseverancia. } \\
\text { Sobriedad, confianza. } \\
\text { honradez, justicia. } \\
\text { Responsabilidad. }\end{array}$ & $\begin{array}{l}\text { Integralidad, } \\
\text { reconocimiento: } \\
\text { respeto, integridad, } \\
\text { generosidad. } \\
\text { empatía, autoestima, } \\
\text { comprensión. }\end{array}$ & $\begin{array}{l}\text { Confianza, compañerismo, } \\
\text { amabilidad: } \\
\text { respeto, interés, cariño, } \\
\text { sensibilidad. }\end{array}$ & \\
\hline 8 & $\begin{array}{l}\text { Relación ética y } \\
\text { productividad }\end{array}$ & $\begin{array}{l}\text { Un mal trabajo es inmoral e } \\
\text { ineficaz y en definitiva no es } \\
\text { productivo. }\end{array}$ & $\begin{array}{l}\text { Las personas son las que } \\
\text { determinan el logro de los } \\
\text { objetivos, a un mejor trato } \\
\text { personal más productivo. }\end{array}$ & $\begin{array}{l}\text { El cimiento verdadero para } \\
\text { el éxito es la gente. }\end{array}$ & $\begin{array}{l}\text { La falta de ética implica } \\
\text { incremento en la } \\
\text { complejidad organizativa, } \\
\text { generando dificultades y } \\
\text { deterioro del proceso } \\
\text { organizativo. }\end{array}$ \\
\hline 9 & $\begin{array}{l}\text { Factores clave } \\
\text { para lograr una }\end{array}$ & $\begin{array}{l}\text { Constancia y perseverancia } \\
\text { en la repetición de los actos } \\
\text { éticos. } \\
\text { Generar motivación, sentido } \\
\text { de la felicidad. }\end{array}$ & $\begin{array}{l}\text { Asumir desde la gerencia un } \\
\text { liderazgo ético. } \\
\text { Dar claridad en las reglas y } \\
\text { transparencia en las } \\
\text { conductas. } \\
\text { La ética como un asunto de } \\
\text { inteligencia empresarial. }\end{array}$ & $\begin{array}{l}\text { Concentrar esfuerzos en la } \\
\text { satisfacción, la felicidad, el } \\
\text { sentido de bondad y la } \\
\text { realización en el trabajo. }\end{array}$ & $\begin{array}{l}\text { Coordinación por } \\
\text { identificación de criterios } \\
\text { entre superior e inferior. } \\
\text { Unidad doctrinal entre los } \\
\text { individuos respecto a un } \\
\text { proyecto común a la } \\
\text { corporación. }\end{array}$ \\
\hline & $\begin{array}{l}\text { o Beneficios de la } \\
\text { ética en la } \\
\text { empresa }\end{array}$ & $\begin{array}{l}\text { Procesos de innovación, } \\
\text { minimizando riesgos. } \\
\text { Se aprende a ser enseñado. }\end{array}$ & $\begin{array}{l}\text { Decisiones justas. } \\
\text { Liderazgo eficiente. }\end{array}$ & $\begin{array}{l}\text { Actuación basada en } \\
\text { valores. } \\
\text { Preocupación humana en } \\
\text { los otros. }\end{array}$ & $\begin{array}{l}\text { Conjunto de valores que } \\
\text { constituyen una identidad } \\
\text { común. } \\
\text { Personal integrado y } \\
\text { motivado. }\end{array}$ \\
\hline & $\begin{array}{l}1 \text { Instrumentos } \\
\text { éticos }\end{array}$ & $\begin{array}{l}\text { Entrenamiento en } \\
\text { comportamientos éticos. }\end{array}$ & $\begin{array}{l}\text { Treinta y cinco } \\
\text { instrumentos. }\end{array}$ & N. A. & N. A. \\
\hline
\end{tabular}

Fuente: Autores 